\title{
Measurement of tissue optical properties and modeling of optimal light delivery for tumor treatment
}

\section{Lihong V. Wang, Guillermo Marquez, Robert E. Nordquist, Wei R. Chen}

Lihong V. Wang, Guillermo Marquez, Robert E. Nordquist, Wei R. Chen, "Measurement of tissue optical properties and modeling of optimal light delivery for tumor treatment," Proc. SPIE 3254, Laser-Tissue Interaction IX, (13 May 1998); doi: 10.1117/12.308181

SPIE Event: BiOS '98 International Biomedical Optics Symposium, 1998, San Jose, CA, United States 


\title{
Measurement of tissue optical properties and modeling of optimal light delivery for tumor treatment
}

\author{
Lihong V. Wang, Ph.D. ${ }^{a}$ \\ Guillermo Marquez, B. S. \\ Optical Imaging Laboratory, Biomedical Engineering Program \\ Texas A\&M University, College Station, Texas 77843-3120, USA \\ Robert E. Nordquist, Ph. D. \\ Wound Healing of Oklahoma, Inc. \\ 3939 N. Walnut Street, Oklahoma City, OK 73105 \\ Wei R. Chen, Ph. D. \\ Oklahoma School of Science and Mathematics \\ 1141 North Lincoln Boulevard, Oklahoma City, OK 73104 \\ Department of Physics and Astronomy, University of Oklahoma, Norman, OK 73109
}

\begin{abstract}
Oblique-incidence reflectometry was used to measure the optical properties of rat tumors with injected absorption-enhancement dye. The measured optical properties were used to model light delivery into the tissues for optimal therapeutic effects. The goal was to efficiently deliver the maximum amount of optical power into buried tumors being treated while avoiding potential damage to normal tissue caused by strong optical power deposition underneath the tissue surface illuminated by the laser beam. The distribution of power deposition was simulated for single beam delivery and multiple beam delivery as well. The simulated results showed that with an appropriate dye enhancement and an optimal laser delivery configuration, a high selectivity for laser treatment of tumors could be achieved.
\end{abstract}

\section{KEY WORDS}

Monte Carlo, light delivery, optical therapy, turbid media, biological tissues.

(a) Corresponding author. E-mail: LWang@tamu.edu. URL: http://biomed.tamu.edu/ lw. 


\section{INTRODUCTION}

Laser-tissue interactions and their therapeutic applications is a fast growing research area. Biological tissues are turbid media, in which light attenuates rapidly because of light absorption augmented by strong light scattering. As a consequence, deeply buried tumors usually receive much less optical power than the subsurface normal tissues, which hampers efficacious optical treatment of tumors. By maximizing the power delivered to the target tumors while avoiding damaging subsurface normal tissues, laser treatments may be improved in several aspects. First, the absorption coefficient of tumors may be significantly increased by infusing dyes into the tumors. Secondly, the wavelength of the laser light may be selected to maximize the ratio between the absorption of the tumor and that of the surrounding normal tissue. Thirdly, the light delivery scheme may be optimized to maximize the power absorption by the target tumors. We will concentrate on the third approach in this paper.

In our work reported in this paper, the optical properties of rat tumors were measured with oblique-incidence reflectometry and Monte Carlo simulations were used to investigate the effect of multiple beam delivery compared with single beam delivery under the condition that the location of the buried tumor was known and the absorption of the tumor was enhanced. Compared with our previous work along this line, ${ }^{1}$ this paper covers more recent work based on the actual optical properties of tumors.

\section{METHOD}

The optical properties of tumors with photosensitizer were measured using oblique-incidence reflectometry. ${ }^{2-4}$ The tumor model was the DMBA-4 metastatic mammary tumor in Wistar Furth female rats. Naive rats ( 6 to 8 weeks) were inoculated with 100,000 viable tumor cells at both inguinal areas. The tumor cells were injected subcutaneously into the fat pads. Within ten days, the primary tumors became palpable and the metastases to axillary areas through lymphatics appeared within 20 days.

The measurement was performed when the primary tumors reached a size of 4 to $8 \mathrm{~cm}^{3}$, and the metastases 0.4 to $0.8 \mathrm{~cm}^{3}$. Following sedation, the hair on the skin overlying the tumors was removed. For dye-enhanced absorption, $200 \mathrm{ml}$ of $0.25 \%$ indocyanine green (ICG) solution was injected into the center of the tumors 2 hours prior to measurement. The tumor was exposed and measured using a $3 \mathrm{~mm}$ probe based on oblique-incidence reflectometry. The measured absorption and reduced scattering coefficient of the tumor at the $805 \mathrm{~nm}$ wavelength were used as input parameters for the subsequent Monte Carlo simulations.

Monte Carlo simulations of light transport in tissues have been implemented previously for simple tissue geometry. ${ }^{5-9}$ To compute light distributions according to the tissue geometry and optical properties, including refractive index $n$, absorption coefficient $\mu_{a}$, scattering coefficient $\mu_{s}$, 
and anisotropy factor $\mathrm{g}$, we have written a Monte Carlo program in $\mathrm{C}$ for tissues with buried objects. We used the delta-scattering technique ${ }^{10}$ for photon tracing to greatly simplify the algorithm because this technique allows a photon packet to be traced without directly dealing with photon crossings of interfaces between different types of tissues. This technique can be used only for refractive-index-matched tissues, although it allows the ambient clear media (e.g., air) and the tissue to have mismatched refractive indices.

We assume that the tissue system has multiple tissue types with identical refractive indices. The interaction coefficient of the $i$ th tissue type, defined as the sum of $\mu_{\mathrm{a}}$ and $\mu_{\mathrm{s}}$, is denoted by $\mu_{\mathrm{i}}$. The technique is briefly summarized as follows.

1. Define a majorant interaction coefficient $\mu_{m}$, where $\mu_{m} \geq \mu_{i}$ for all $i$.

2. Select a step size $\mathbf{R}$ between two consecutive interactions based on the majorant interaction coefficient,

$$
\mathrm{R}=-\ln (\xi) / \mu_{\mathrm{m}},
$$

where $\xi$ is a uniformly distributed random number between 0 and $1(0<\xi \leq 1)$. Then, determine the tentative next collision site $\mathbf{r}_{\mathrm{k}}{ }^{\prime}$ by:

$$
\mathbf{r}_{\mathrm{k}}{ }^{\prime}=\mathbf{r}_{\mathrm{k}-1}+\mathrm{R} \mathbf{u}_{\mathrm{k}-1} \text {, }
$$

where $\mathbf{r}_{\mathbf{k}-1}$ is the current site and $\mathbf{u}_{\mathrm{k}-1}$ is the direction of the flight.

3. Play a rejection game:

a. Get a random number $\eta$, which is a uniformly distributed random number between 0 and $1(0<\eta \leq 1)$.

a. If $\eta \leq \mu_{i}\left(r_{k}{ }^{\prime}\right) / \mu_{m}$, i.e., with a probability of $\mu_{i}\left(r_{k}{ }^{\prime}\right) / \mu_{m}$, accept this point as a real interaction site $\left(\mathbf{r}_{\mathbf{k}}=\mathbf{r}_{\mathbf{k}}{ }^{\prime}\right)$.

b. Otherwise, do not accept $\mathbf{r}_{\mathbf{k}}{ }^{\prime}$ as a real interaction site but select a new path starting from $\mathbf{r}_{k}{ }^{\prime}$ with the unchanged direction $\mathbf{u}_{\mathrm{k}-1}$ (i.e., set $\mathbf{r}_{\mathrm{k}-1}=\mathbf{r}_{\mathrm{k}}{ }^{\prime}$ and return to Step 2).

The treatment of photon tracing after step 3 is similar to that in Ref. 9 and will not be repeated here.

The validity can be easily understood by introducing an imaginary interaction event that changes neither the weight nor the direction of the photon. This definition implies that such imaginary interactions are not physically observable, i.e., they can be introduced with any interaction coefficient at any point. We may assume that the majorant interaction coefficient $\mu_{m}$ is a sum of the real $\mu_{\mathrm{re}}$ and imaginary $\mu_{\mathrm{im}}$ interaction coefficients, where the real interaction coefficient is $\mu_{\mathrm{re}}$ is $\mu_{\mathrm{i}}\left(\mathbf{r}_{\mathrm{k}}{ }^{\prime}\right)$. In the procedure outlined above, a fraction of the interactions,

$$
1-\mu_{\mathrm{re}} / \mu_{\mathrm{m}}=\mu_{\mathrm{im}} / \mu_{\mathrm{m}}
$$

are imaginary interactions. From another point of view, it is easy to see that on the average, for every $\mu_{\mathrm{m}}$ total interactions, there will be $\mu_{\mathrm{re}}$ interactions accepted as real interactions. The mean free 
path for the majorant interactions in the delta-scattering method is $1 / \mu_{m}$, and the mean free path for the real interactions in the direct method is $1 / \mu_{\mathrm{rc}}$. Therefore, the photon will move to the correct interaction site using the delta-scattering technique as it would using the direct method because

$$
\mu_{\mathrm{m}}\left(1 / \mu_{\mathrm{m}}\right)=\mu_{\mathrm{re}}\left(1 / \mu_{\mathrm{re}}\right),
$$

where the left-hand side means the average distance traveled by the photon packet with $\mu_{\mathrm{m}}$ total steps or with $\mu_{\mathrm{re}}$ real interactions in the delta-scattering method, and the right-hand side means the average distance traveled with $\mu_{\mathrm{re}}$ real interactions in the direct method.

During the tracing of each weighted photon, ${ }^{9}$ the light absorption, reflection, or transmission were correspondingly scored into different arrays according to the spatial positions of the photon. Multiple photons are traced to achieve an acceptable statistical variation. For this study, 100,000 photons were traced.

This Monte Carlo program was used to simulate power deposition for tissue configurations as shown in Fig. 1. Fig. 1(a) shows a single beam delivery scheme to a tissue slab with a tumor buried in the center. The lateral dimensions in the xy-plane was considered optically infinite, i.e., much greater than the penetration depth of light. Fig. 1(b) illustrates a multiple beam delivery scheme to a tissue cube with a tumor buried in the center.
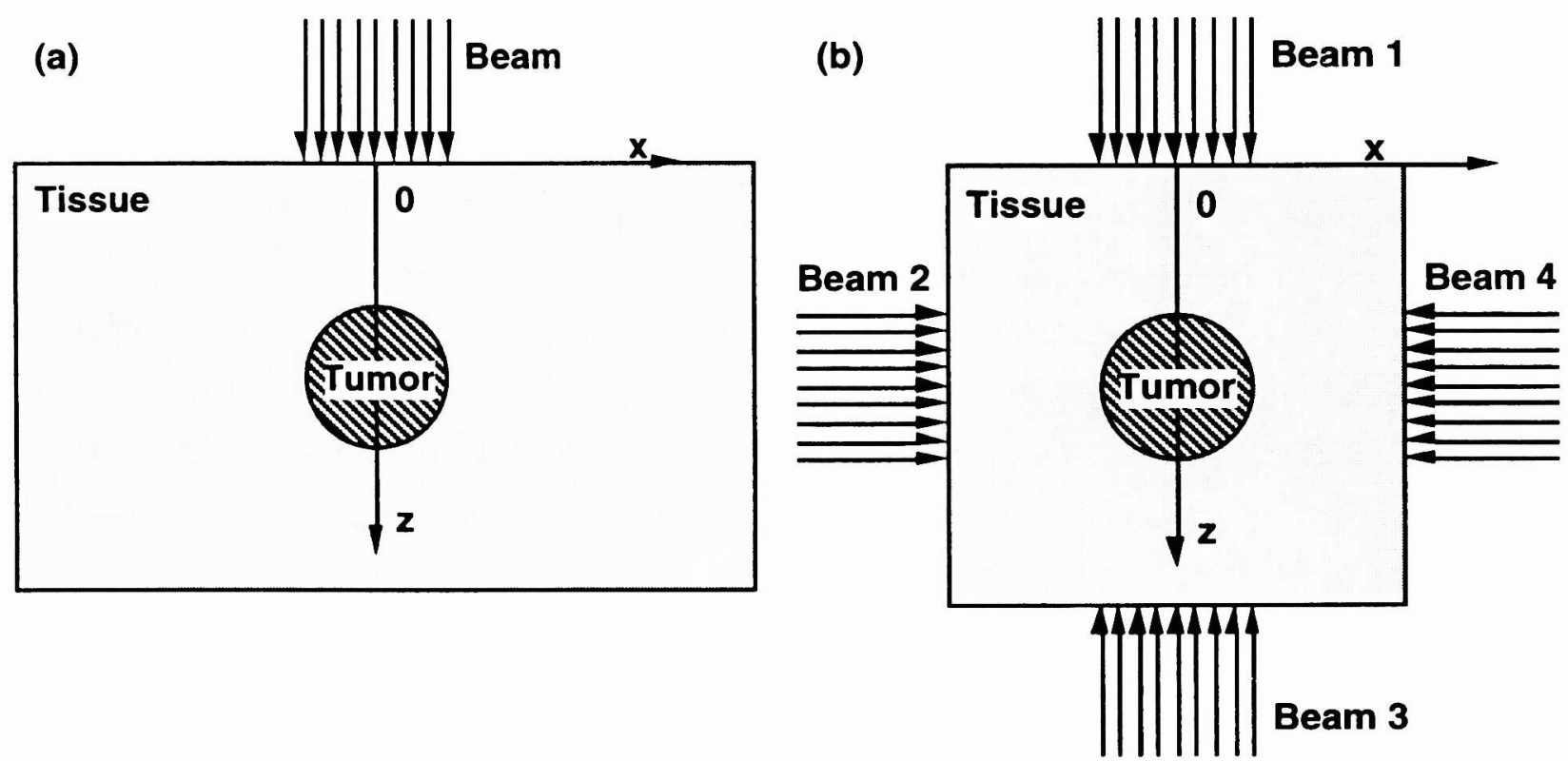

Fig. 1. Configurations of the biological tissue and laser beams: (a) single beam delivery to a wide tissue slab and (b) multiple beam delivery to a tissue cube. The tumor was a sphere that was centered in the background tissue and aligned with the center of the laser beam. In both cases, the optical properties of the background tissue were: absorption coefficient $\mu_{\mathrm{a}}=0.1 \mathrm{~cm}^{-1}$, scattering coefficient $\mu_{\mathrm{s}}=100 \mathrm{~cm}^{-1}$, anisotropy $\mathrm{g}=0.9$. The optical properties of the tumor were: variable $\mu_{\mathrm{a}}, \mu_{\mathrm{s}}=140 \mathrm{~cm}^{-1}, \mathrm{~g}=0.9$. The diameter of the tumor was $1 \mathrm{~cm}$. The thickness of the tissue slab in (a) and the side of the tissue cube in (b) were both $3 \mathrm{~cm}$. 


\section{RESULTS and CONCLUSIONS}

The absorption coefficient and reduced scattering coefficient of the tumor containing ICG were measured to be $5 \mathrm{~cm}^{-1}$ and $14 \mathrm{~cm}^{-1}$, respectively. Assuming the scattering anisotropy $\mathrm{g}$ of the tumor was 0.9 , we calculated the scattering coefficient to be $140 \mathrm{~cm}^{-1}$. For energy deposition far from the source, the choice of scattering anisotropy $g$ was not important as long as the reduced scattering coefficient was conserved because the energy transport had entered diffusion regime. The optical properties of the normal tissue surrounding the tumor were not measured in this experiment because there was not enough tissue to measure on the rats. We used a typical set of optical properties for the surrounding tissue for the model, where the absorption coefficient, the scattering coefficient, and anisotropy were $0.1 \mathrm{~cm}^{-1}, 100 \mathrm{~cm}^{-1}$, and 0.9 , respectively.

An optimal radius of the laser beam was calculated based on the following expression ${ }^{1}$

$$
r_{o}=\sqrt{\delta\left(2 d_{t}-2 L_{i}{ }^{\prime}+\delta\right)}
$$

where $d_{t}$ was the distance between the center of the tumor and the upper tissue surface, and $\delta$ was the penetration depth. The calculated optimal radius was $1.4 \mathrm{~cm}$, which was used for this modeling.

The energy deposition in tissue was modeled using various values of absorption coefficient for the tumor for single-beam delivery (Fig. 2). As can be seen, the energy deposition in the tumor became comparable with the subsurface energy deposition when the absorption coefficient of the tumor was raised to $1 \mathrm{~cm}^{-1}$. With an further increased absorption coefficient for the tumor, the center and bottom of the tumor received relatively less light because more light was absorbed by the top of the tumor.

The energy deposition in tissue was modeled using various values of absorption coefficient for the tumor for multiple-beam delivery as well (Fig. 3). As the absorption coefficient of the tumor was increased, the surface of tumor received more and more light energy. But the center of the tumor received less and less light energy because much of the light was absorbed by the tumor shell and did not penetrate into the center. Therefore, the surface of the tumor may be killed immediately during phototherapeutics. The center of the tumor may be "starved" to death later because the blood supply to the center of the tumor was interrupted around the shell. The fourbeam delivery yielded a much more homogeneous and stronger energy deposition into the tumor than the single-beam delivery without increasing the subsurface energy deposit. The energy deposition on the tumor surface was even stronger than that under the tissue surface when the absorption coefficient of the tumor was enhanced enough, which was highly desirable in optical therapeutics. 
(a) $\mu_{\mathrm{a}}=0.1 \mathrm{~cm}^{-1}$
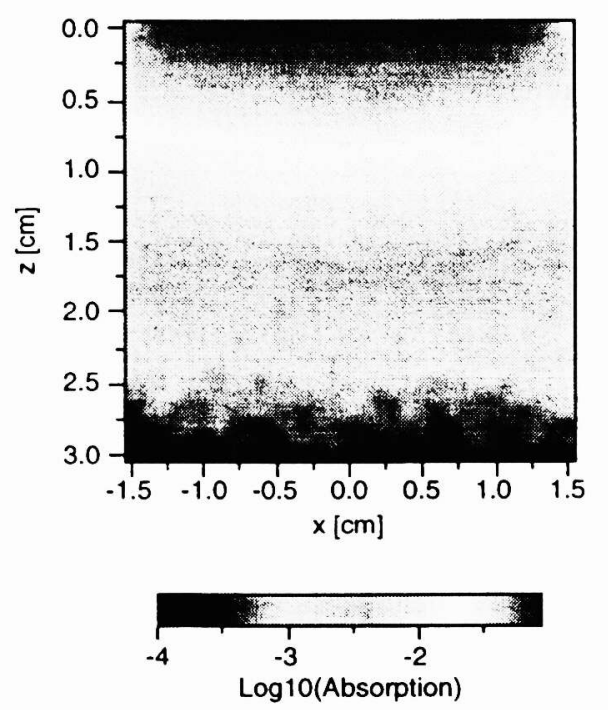

(c) $\mu_{\mathrm{a}}=3 \mathrm{~cm}^{-1}$
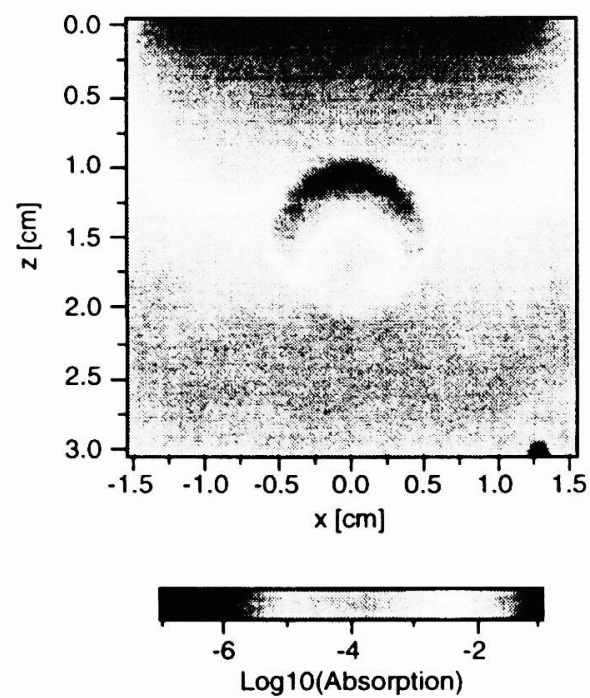

(b) $\mu_{\mathrm{a}}=1 \mathrm{~cm}^{-1}$
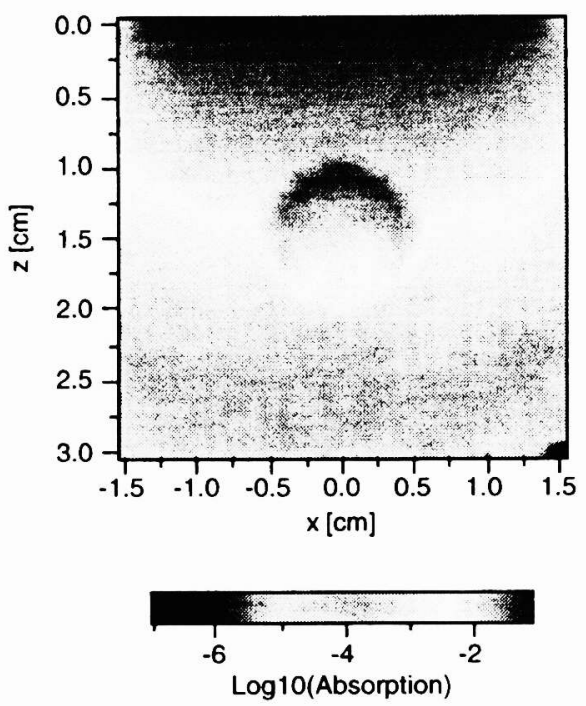

(d) $\mu_{\mathrm{a}}=5 \mathrm{~cm}^{-1}$
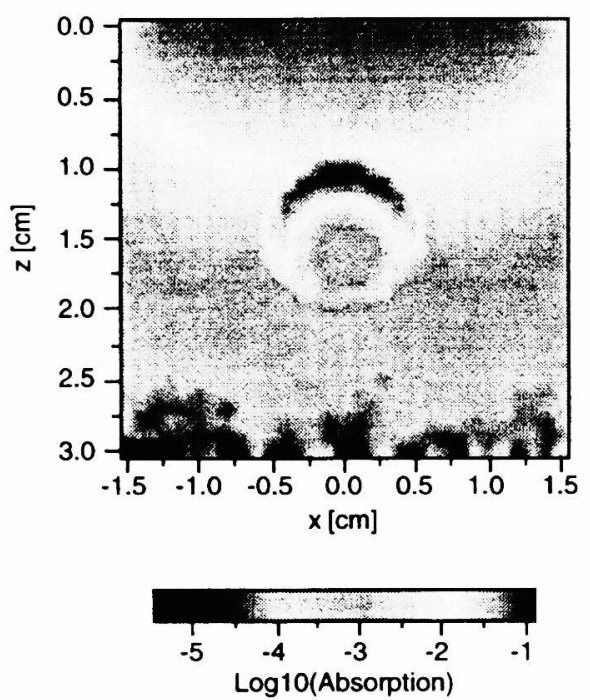

Fig. 2. False-color plots of the deposited power density distribution in $\mathrm{W} / \mathrm{cm}^{3}$ in the tissue in $\log$ scale for one-beam delivery when the absorption coefficient of the tumor was set to (a) $0.1 \mathrm{~cm}^{-}$, (a) $1 \mathrm{~cm}^{-1}$, (a) $3 \mathrm{~cm}^{-1}$, (a) $5 \mathrm{~cm}^{-1}$. The total power of the incident laser beam $P_{s}$ was set to $1 \mathrm{~W}$. 
(a) $\mu_{\mathrm{a}}=0.1 \mathrm{~cm}^{-1}$
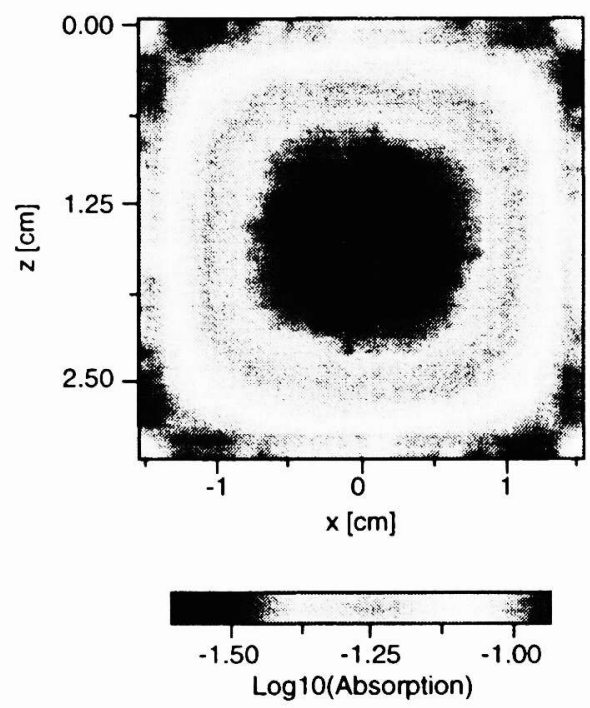

(c) $\mu_{\mathrm{a}}=3 \mathrm{~cm}^{-1}$
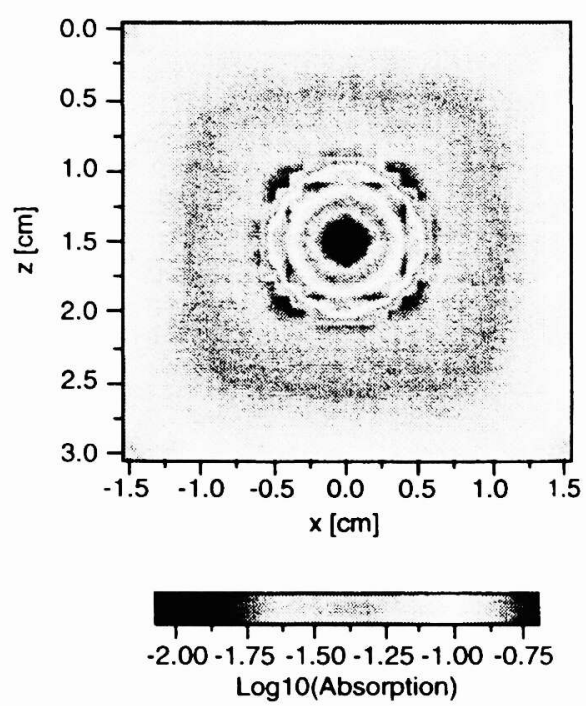

(b) $\mu_{\mathrm{a}}=1 \mathrm{~cm}^{-1}$
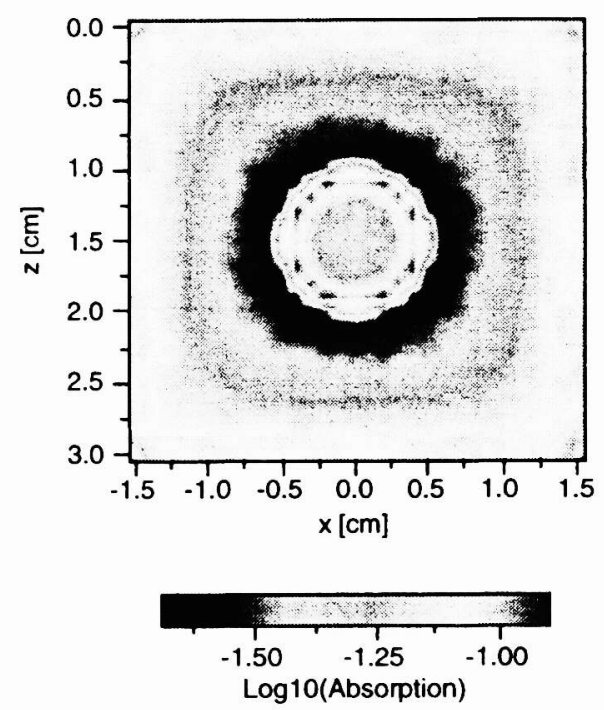

(d) $\mu_{\mathrm{a}}=5 \mathrm{~cm}^{-1}$
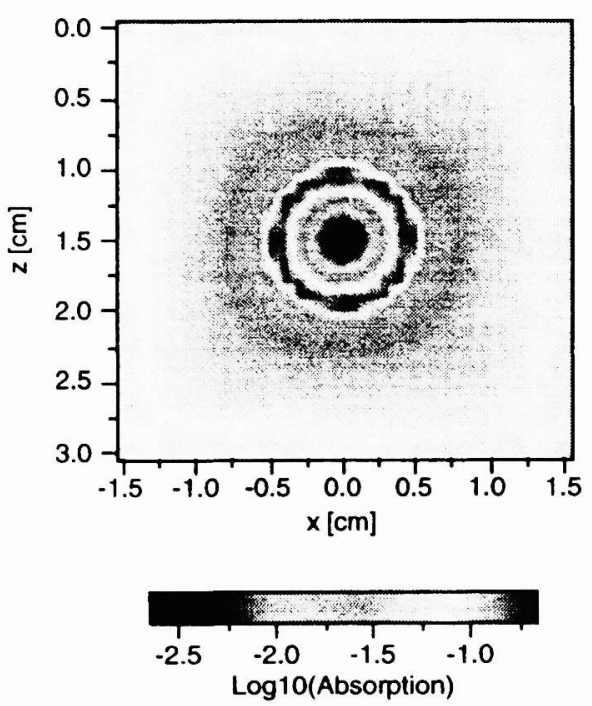

Fig. 3. False-color plots of the deposited power density distribution in $\mathrm{W} / \mathrm{cm}^{3}$ in the tissue in $\log$ scale for four-beam delivery when the absorption coefficient of the tumor was set to (a) $0.1 \mathrm{~cm}^{-}$, (a) $1 \mathrm{~cm}^{-1}$, (a) $3 \mathrm{~cm}^{-1}$, (a) $5 \mathrm{~cm}^{-1}$. The total power of each incident laser beam $P_{s}$ was set to $1 \mathrm{~W}$. 


\section{SUMMARY}

Oblique-incidence was able to measure optical properties of biological tissues in vivo. Local administration of ICG was found to enhance the absorption coefficient of tumors significantly. Monte Carlo simulations were proved to be a flexible and powerful tool in studying light transport in heterogeneous biological tissues. Four-beam delivery was found superior to single-beam delivery. In the four-beam delivery scheme, the tumor with an ICG-enhanced absorption coefficient was found to absorb more light energy than the surface of the surrounding tissue. However, the center of the tumor received little light and is expected to be killed indirectly in response to lack of blood supply. These results provide guidelines for various laser therapeutics including photothermal therapies, photomechanical therapies, and noninvasive photosensitizerassisted laser therapies of deep tumors.

\section{ACKNOWLEDGMENT}

The project was sponsored in part by The Whitaker Foundation grant and the National Institutes of Health grant R29 CA68562 and R01 CA71980. 


\section{GLOSSARY}

\begin{tabular}{|c|c|}
\hline $\begin{array}{l}\text { Absorption coefficient } \\
{\left[\mu_{\mathrm{a}}, \mathrm{cm}^{-1}\right]}\end{array}$ & The probability of photon absorption per unit infinitesimal pathlength. \\
\hline Anisotropy $[\mathrm{g}]$ & $\begin{array}{l}\text { The average of the cosine value of the deflection angle by single } \\
\text { scattering. }\end{array}$ \\
\hline $\begin{array}{l}\text { Diffusion constant } \\
{[\mathrm{D}, \mathrm{cm}]}\end{array}$ & $\begin{array}{l}\text { Linking the gradient of light fluence, } \nabla \phi \text {, and light current, F, (Fick's } \\
\text { law), i.e., } F=-D \nabla \phi \text {. }\end{array}$ \\
\hline $\begin{array}{l}\text { Effective attenuation } \\
\text { coefficient }\left[\mu_{\text {eff }}, \mathrm{cm}^{-1}\right]\end{array}$ & $\begin{array}{l}\text { The decay constant of light fluence far away from light source. } \\
\mu_{\text {eff }}=\sqrt{3 \mu_{\mathrm{a}} / \mathrm{D}} \text {. }\end{array}$ \\
\hline $\begin{array}{l}\text { Interaction coefficient } \\
{\left[\mu_{\mathrm{t}}, \mathrm{cm}^{-1}\right]}\end{array}$ & $\begin{array}{l}\text { The probability of photon interaction per unit infinitesimal pathlength, } \\
\text { where the interaction includes both absorption and scattering. } \mu_{t}=\mu_{a} \\
+\mu_{s} \text {. Sometimes, it is also called total interaction coefficient or total } \\
\text { attenuation coefficient. }\end{array}$ \\
\hline Mean free path $[\mathrm{mfp}]$ & The mean pathlength between interactions, which is $1 / \mu_{\mathrm{t}}$. \\
\hline $\begin{array}{l}\text { Penetration depth }[\delta \\
\mathrm{cm}]\end{array}$ & $\begin{array}{l}\delta=1 / \mu_{\text {eff }} \text { It represents decay constant of the light fluence far from the } \\
\text { source. }\end{array}$ \\
\hline $\begin{array}{l}\text { Reduced scattering } \\
\text { coefficient }\left[\mu_{\mathrm{s}}^{\prime}, \mathrm{cm}^{-1}\right]\end{array}$ & $\begin{array}{l}\mu_{\mathrm{s}}^{\prime}=\mu_{\mathrm{s}}(1-\mathrm{g}) \text {. Sometimes, it is also called transport scattering } \\
\text { coefficient. }\end{array}$ \\
\hline $\begin{array}{l}\text { Scattering coefficient } \\
{\left[\mu_{s}, \mathrm{~cm}^{-1}\right]}\end{array}$ & The probability of photon scattering per unit infinitesimal pathlength. \\
\hline $\begin{array}{l}\text { Transport interaction } \\
\text { coefficient }\left[\mu_{\mathrm{t}}^{\prime}, \mathrm{cm}^{-1}\right]\end{array}$ & $\mu_{\mathrm{t}}^{\prime}=\mu_{\mathrm{a}}+\mu_{\mathrm{s}}^{\prime}$ \\
\hline $\begin{array}{l}\text { Transport mean free } \\
\text { path [mfp'] }\end{array}$ & $1 / \mu_{\mathrm{t}}^{\prime}$ \\
\hline
\end{tabular}




\section{REFERENCES}

1. L.-H. Wang, R. E. Nordquist, and W. R. Chen, "Optimal beam size for light delivery to absorption-enhanced tumors buried in biological tissues and effect of multiple beam delivery: a Monte Carlo study," Appl. Opt. 36, 8286-8291 (1997).

2. L.-H. Wang and S. L. Jacques, "Use of a laser beam with an oblique angle of incidence to measure the reduced scattering coefficient of a turbid medium," Appl. Opt. 34, 2362-2366 (1995).

3. S.-P. Lin, L.-H. Wang, S. L. Jacques, and F. K. Tittel, "Measurement of tissue optical properties using oblique incidence optical fiber reflectometry," Appl. Opt. 36, 136-143 (1997).

4. G. Marquez and L.-H. Wang, "White light oblique incidence reflectometer for measuring absorption and reduced scattering spectra of tissue-like turbid media," Optics Express 1, 454460 (1997).

5. B. C. Wilson and G. A. Adam, "Monte Carlo model for the absorption and flux distributions of light in tissue," Med. Phys. 10, 824-830 (1983).

6. S. T. Flock, B. C. Wilson, D. R. Wyman, and M. S. Patterson, "Monte-Carlo modeling of light-propagation in highly scattering tissues $\mathrm{I}$ : model predictions and comparison with diffusion-theory," IEEE Trans. Biomed. Eng. 36, 1162-1168 (1989).

7. S. A. Prahl, M. Keijzer, S. L. Jacques, and A. J. Welch, "A Monte Carlo model of light propagation in tissue," Proc. Soc. Photo-Opt. Instrum. Eng. IS 5, 102-111 (1989).

8. S. L. Jacques and L.-H. Wang, "Monte Carlo modeling of light transport in tissues," in Optical Thermal Response of Laser Irradiated Tissue, edited by A. J. Welch and M. J. C. van Gemert (Plenum Press, New York, 1995), pp. 73-100.

9. L.-H. Wang, S. L. Jacques, and L.-Q. Zheng, "MCML - Monte Carlo modeling of photon transport in multi-layered tissues," Computer Methods and Programs in Biomedicine 47, 131146 (1995). Note: The simulation software package can be downloaded from http://biomed.tamu.edu/ lw.

10. I. Lux and L. Koblinger, Monte Carlo Particle Transport Methods: Neutron and Photon Calculations (CRC Press, Boca Raton, 1991). 